\title{
Research on Optimal MPPT of Photovoltaic System Based on Disturbance Observation Method
}

\author{
Jianhua Deng ${ }^{1}$, Yanping Wang, ${ }^{2, *}$ \\ \{djhisgood@yeah.net ${ }^{1}$,wangyp@dlpu.edu.cn² ${ }^{2}$, \\ ${ }^{1}$ Department of Information Science and Engineering Dalian Polytechnic University Dalian, P. R. \\ China \\ ${ }^{2}$ Dalian Key Laboratory of Smart Micro-grid and Green Recycling Industry, Liaoning province
}

\begin{abstract}
The biggest problem with the photovoltaic power generation system is that the energy utilization rate is too low due to the instability of its power. Therefore, a reasonable method must be developed to track its maximum power so that the photovoltaic cell can output the maximum power at all times. Compared with the large oscillation at the maximum power point of the traditional disturbance observation method, an improved disturbance observation method with PID control duty cycle is proposed. The duty cycle of the boost circuit is controlled by the PID algorithm and the disturbance step length of the $P-D$ characteristic is changed. Match the load impedance with the input impedance of the photovoltaic cell, so the photovoltaic cell outputs the maximum power, increasing the tracking efficiency.
\end{abstract}

Keywords: Photovoltaic system; maximum power tracking; PID control

\section{Introduction}

Since the output power of photovoltaic cells will change with changes in the external environment and load, it must be tracked and controlled so that photovoltaic cells always output at maximum power. At present, the commonly used maximum power tracking (MPPT) methods for photovoltaic systems include constant voltage method, disturbance observation method, conductance increment method, etc. [1-3]. Literature [3] proposes a tracking method that directly perturbs the duty cycle. Literature [9] verifies the effectiveness of directly perturbing the duty cycle through simulation, but there is a certain deviation, which is different from the initial value of the duty cycle.

Select the relevant, this article introduces a method based on the perturbation observation method, which is an optimized method in the literature [8]. The general process of this method is to add an MPPT module between the photovoltaic cell and the load.

The function of this module is to sample the duty cycle and output power, and then compare the power of equidistant points on both sides of the duty cycle, and finally determine the direction of the disturbance.

* Corresponding Author: Yanping Wang

Fund Project: Dalian Science and Technology Innovation Fund (2020JJ26GX029)

The Natural Science Foundation of Educational Department of Liaoning Province (Grant: J2020053) 
The function of this module is to sample the duty cycle and output power, and then compare the power of equidistant points on both sides of the duty cycle, and finally determine the direction of the disturbance. Compared with the traditional disturbance observation method, the tracking efficiency is improved. Effectively avoid the oscillation phenomenon of power tracking. By controlling the duty cycle of the switching element in the Boost circuit, the load impedance is matched with the input impedance of the photovoltaic cell, so the maximum power is output and the photovoltaic cell is boosted at the same time.

According to the characteristics of the photovoltaic cell, the simulink model is established, and the improved disturbance observation method is combined to simulate it, and the result of MPPT is obtained.

\section{Photovoltaic cell modeling and output characteristic simulation}

\subsection{Photovoltaic cell equivalent circuit}

There are three types of equivalent circuits for photovoltaic cells [4]. The more commonly used equivalent circuits are shown in Figure 1.

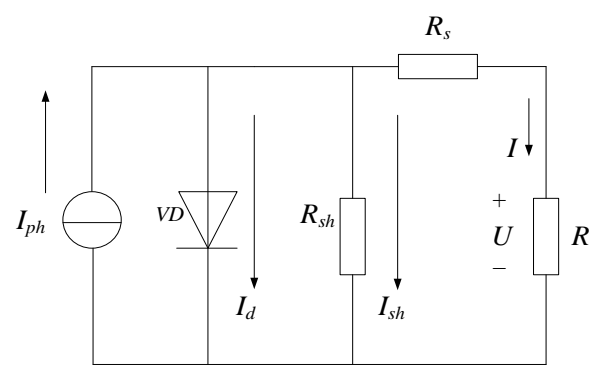

Fig. 1.Solar photovoltaic cell equivalent circuit.

The $I-U$ characteristic curve of an ideal photovoltaic cell is obtained by subtracting the current of the diode from the photovoltaic cell photocurrent, but the photovoltaic cell in the experiment should consider the actual internal loss of the battery. Therefore, the model shown in the above [1]figure not only considers the series resistance $R_{s}$, the resistance value is generally low, which means the loss resistance of the line [5], but also considers the parallel resistance $R_{s h}$, which means the bypass resistance, and the resistance value is generally higher., The order of magnitude is $10^{3} \Omega, I_{s h}$ is the leakage current caused by the process, $I_{p h}$ is the photogenerated current proportional to the light intensity, $I$ is the output current, and $I_{d}$ is the diode saturation current [6]. According to the circuit diagram, Kirchhoff's theorem can be obtained:

$$
I=I_{P h}-I_{d}-I_{s h}
$$

The expression of $I_{d}$ and $I_{s h}$ in formula (1) is (2).

$$
I_{d}=I_{0}\left\{\exp \left[\frac{q\left(U+I \times R_{S}\right)}{n K T}\right]-1\right\} \quad I_{s h}=\frac{U+I R_{S}}{R_{S h}}
$$

In the formula, $I_{0}$ is the saturated reverse current, $K$ is Boltzmann's constant, and the value is $1.38 \times 10^{-23} \mathrm{~J} / \mathrm{K} ; n$ is the ideal factor of the diode, usually about 1.3 , and $q$ is the charge of the 
electron, which is $1.6 \times 10^{-19} \mathrm{C}$.

Substituting (2) into (1) can obtain the characteristic expression (3) of photovoltaic cells:

$$
I=I_{p h}-I_{0}\left\{\exp \left[\frac{q\left(U+I \times R_{S}\right)}{n K T}\right]-1\right\}-\frac{U+I R_{S}}{R_{s h}}
$$

Since the value of the bypass resistor $R_{s h}$ is particularly large, the bypass current $I_{s h}$ is small enough to be ignored, so the above equation can be rewritten as (4).

$$
I=I_{p h}-I_{0}\left\{\exp \left[\frac{\mathrm{q}\left(U+I \times R_{s}\right)}{n K T}\right]-1\right\}
$$

Because $R_{s}$ in the equivalent circuit diagram of the photovoltaic cell is much smaller than the forward conduction resistance of the diode, it can be approximated as $I_{p h}=I_{s c}$.

According to the $I-U$ relationship of the photovoltaic cell, the output characteristic equation can be obtained. At the same time, the engineering mathematical model is established, and $I_{0}=A_{1} I_{s c}, \frac{n K T}{q}=A_{2} U_{o c}$.Available (5):

$$
I=I_{s c}-A_{1} I_{s c}\left\{\exp \left[\frac{U}{U_{o c} A_{2}}\right]-1\right\}
$$

Solve $A_{1}$ and $A_{2}$ by formula (5):

$$
\begin{gathered}
A_{1}=\left(1-\frac{I_{m}}{I_{s c}}\right) \exp \left(\frac{-U_{m}}{A_{2} U_{o c}}\right) \\
A_{2}=\left(\frac{U_{m}}{U_{o c}}-1\right)\left[\ln \left(1-\frac{I_{m}}{I_{s c}}\right)\right]^{-1}
\end{gathered}
$$

In practical applications, the short-circuit current of photovoltaic cells under standard conditions (light intensity $S_{c}=1000 \mathrm{~W} / \mathrm{m}^{2}$, battery temperature $T=25$ degrees Celsius) is $I_{s c}$, the open circuit voltage is $U_{o c}$, the maximum power point $U_{m}$, and the maximum power point current $I_{m}$. These parameters are fixed values, so $A_{1}, A_{2}$ can be obtained as a constant. When the temperature or light intensity changes, it is necessary to recalculate the 4 parameters under the new conditions as $I_{s c-n}, I_{m-n}, U_{o c-n}, U_{m-n}$. And recalculate $A_{1-n}, A_{2-n}$, under the new conditions:

$$
\begin{gathered}
\Delta T=T-T_{c} \\
\Delta S=\frac{s}{S_{c}}-1 \\
I_{s c-n}=I_{s c}\left(\frac{S}{S_{c}}\right)(1+a \Delta T) \\
I_{m-n}=I_{m}\left(\frac{S}{S_{c}}\right)(1+a \Delta T) \\
U_{o c-n}=U_{o c}[(1-c \Delta T) \ln (e+b \Delta S)] \\
U_{m-n}=U_{m}[(1-c \Delta T) \ln (e+b \Delta S)]
\end{gathered}
$$

The coefficients in the formula are typical values: $a=0.0025, b=0.5, c=0.00288$. To sum up, build a simulation model in simulink according to the engineering mathematical model of photovoltaic cells as shown in the figure. The parameter settings select the photovoltaic cell model CN-200S on the market: $P_{m}=120 \mathrm{~W}, I_{s c}=7.68 \mathrm{~A}, I_{m}=6.94 \mathrm{~A}, U_{o c}=17.5 \mathrm{~V}, U_{m}=28.8 \mathrm{~V}$. The coefficients $a, b, c$ in the formula are typical values. The model of the photovoltaic cell is shown 
in the Figure 2.

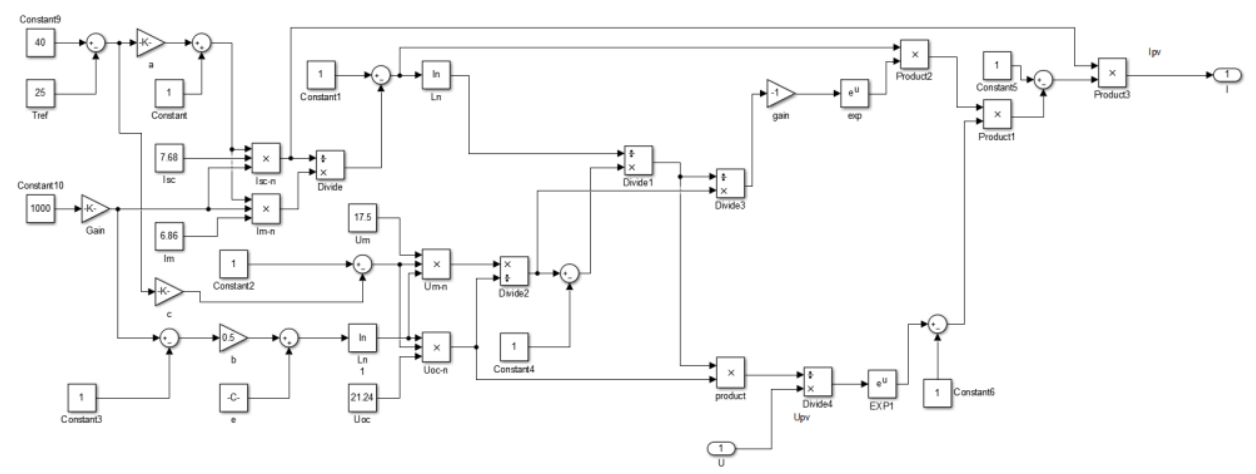

Fig. 2. Photovoltaic cell model.

\subsection{Photovoltaic cell simulation results}

Run the simulation of the photovoltaic cell model, and finally get the output characteristic curve of the photovoltaic cell. As shown Figure 3.
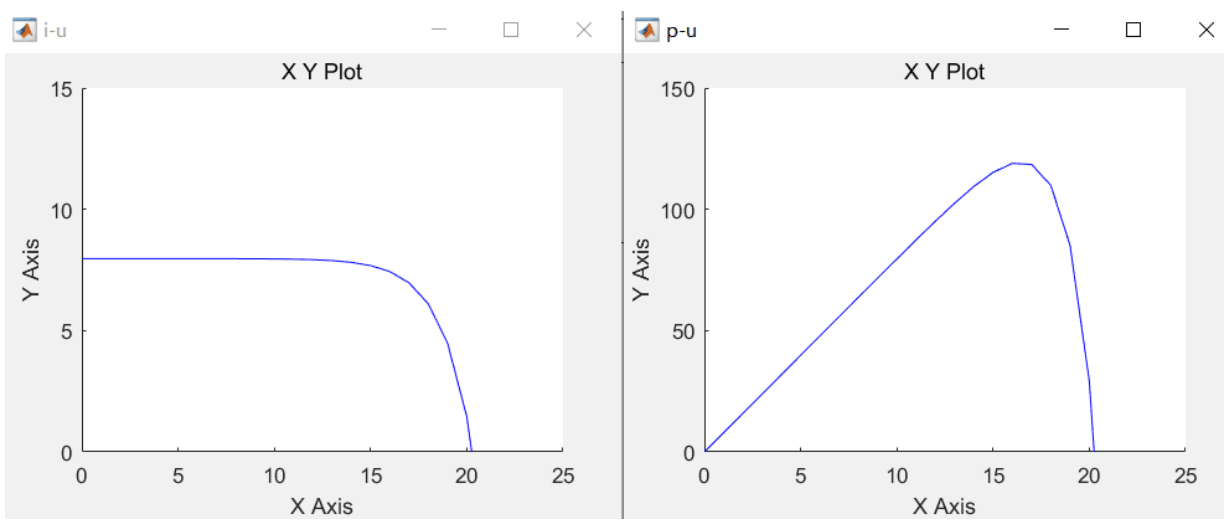

Fig. 3. $U-I, U-P$ characteristic curves of photovoltaic cells.

Figure 4 is the $P-U$ characteristic curve diagram of the output of the photovoltaic cell when the temperature is constant and the light intensity is different. The image shows that the output power of the photovoltaic cell changes with the voltage. Under certain external conditions, the photovoltaic cell has a maximum output power. 


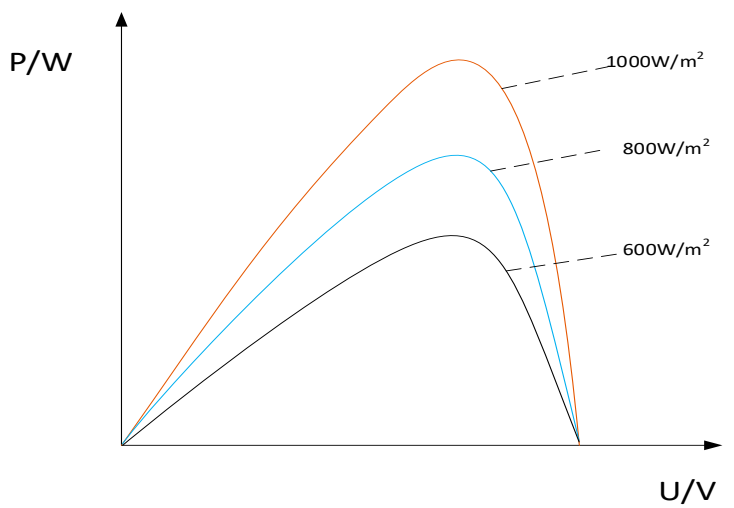

Fig. 4. Photovoltaic cell $P-U$ diagram.

It can be seen from Figure 4 that when the temperature of the photovoltaic cell is constant, as the light intensity increases, the short-circuit current of the photovoltaic cell will increase with the increase of the light intensity; the output power of the photovoltaic cell will increase with the increase of the light intensity. And the maximum output power also increases with the increase of light intensity. It is worth mentioning that their changes are very obvious.

Therefore, we can get a conclusion, that is the power-voltage relationship and the currentvoltage relationship of photovoltaic cells is not a linear relationship, but an obvious non-linear relationship.

At the same time, we can also get that the short-circuit current and output power of photovoltaic cells are greatly affected by the light intensity, and the open circuit voltage is relatively small.

\section{Disturbance Duty Cycle Control Method}

\subsection{Traditional disturbance observation}

During the entire process of photovoltaic power generation grid-connected system, the efficiency of photovoltaic cells is worth considering. According to statistics, the efficiency of photovoltaic cells is only about $10 \%$, which limits the operation of photovoltaic power generation systems to a certain extent. It is known that the output characteristics of photovoltaic cells are affected by light intensity and temperature, and it has been known that the output characteristic curve of photovoltaic cells is not a straight line, so they are in a nonlinear relationship.

When the external environmental conditions change, the output of photovoltaic cells Voltage and current will change, output power will also change, but no matter how it changes, there is only one maximum power point.

The process of traditional disturbance observation method is to artificially apply a disturbance amount $\Delta U$ to the output voltage of the photovoltaic cell, observe the power change, if the output power increases, continue to impose disturbance in this direction, if the power decreases, impose disturbance in the reverse direction. 
When the power is reduced again, it means that it is near the maximum power point, and it continues continuously, and finally the power has been oscillating near the maximum power point. This method is relatively simple to implement, but there will be a lot of power loss at the maximum power point. The traditional disturbance observation method MPPT model is shown in the Figure 5.

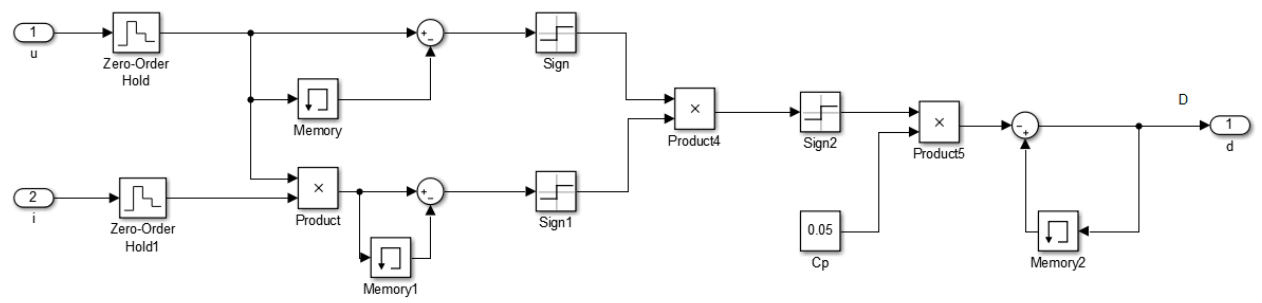

Fig. 5. Traditional disturbance observation method MPPT model.

\subsection{PID disturbance duty cycle observation method}

Because the $P$ - $D$ curve of the photovoltaic system is basically similar to the $P$ - $U$ image, the disturbance observation method is also applicable. This method tracks the power by controlling the duty cycle of the switching element in the Boost circuit. The specific method is as follows $[7]$.

As shown in Figure 6, it is a Boost circuit with a period of $T$ and a duty cycle of $d$. According to the circuit, the relationship between the duty cycle and the voltage can be obtained.

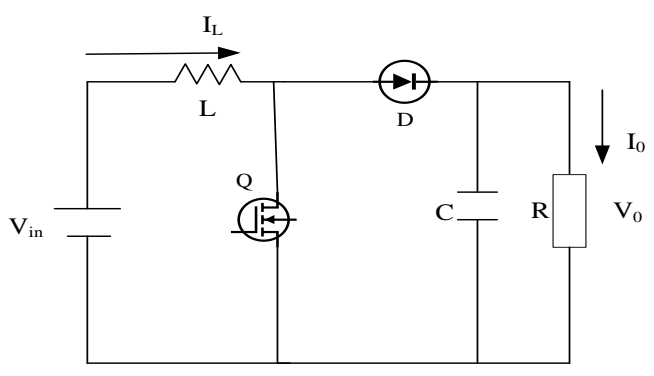

Fig. 6. Boost circuit

When the switching element is turned on, the photovoltaic power supply charges the inductor and the capacitor discharges the resistor. The current that the inductor increases during this process is $\Delta I_{L}=\frac{V_{\text {in }}}{L} d T$.

When the switching element is turned off, the inductor and the photovoltaic power supply both charge the capacitor, and the current reduced by the inductor is $\Delta I_{L}^{\prime}=\frac{V_{i n}-V_{0}}{L} T(1-d)$.

In a cycle, when a continuous current flows through the inductor, the charging current and 
the discharging current of the inductor are equal, so you can get $\Delta I_{L}=\Delta I_{L}^{\prime}$, so $V_{0}=\frac{V_{\text {in }}}{1-d}, I_{0}=$ $I_{L}(1-d)$.

Therefore, the input impedance of the photovoltaic cell can be obtained $R_{i n}=R(1-d)^{2}$, so only the duty cycle $d$ needs to be adjusted so that the input impedance of the photovoltaic cell is equal to the load impedance, and the maximum power can be obtained. Improved tracking efficiency.

\subsection{How to change the duty cycle through PID control}

Track the maximum power of the photovoltaic cell and then output the maximum power. This paper proposes an MPPT strategy based on the perturbation and observation method. The method is to obtain a duty cycle that can be automatically changed through the perturbation observation method, and then PID control the duty cycle Then output the final duty cycle. The specific method is as follows.

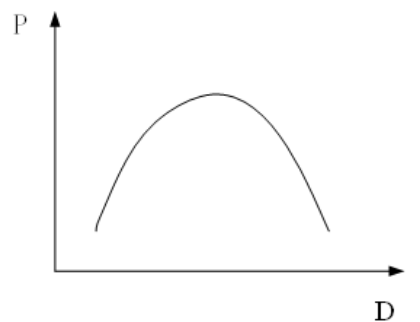

Fig. 7. Photovoltaic cell $P-D$ diagram

The $P-D$ relationship diagram of the photovoltaic cell can be obtained by calculation as shown in the Figure 7, so any point in the figure above indicates that the size of $D$ at a certain moment is $D_{k}$, and then two equidistant points are taken before and after this point.

They are $D_{k-1}, D_{k+1}$ and $D_{k+1}=D_{k}+\Delta D, D_{k-1}=D_{k}-\Delta D$, and finally 9 different situations of three point powers can be obtained according to different positional relationships [8].

Suppose that point $A$ on the $P-D$ diagram is taken as the original operating point, points $B$ and $C$ are two sampling points with the same step distance from $A$, and the power of the three points is $P_{k}$ for point $A$ and $P_{k-1}$ for point $B$. Point $C$ is $P_{k+1}$. Then the direction of duty cycle disturbance can be determined by comparing the power relationship between $A$ and $B, C$ and $A$.

If both comparisons are greater than, it can be determined that the photovoltaic cell is working on the left side of the maximum power point at this time, and the duty cycle should be increased at this time. If both comparisons are less than, it can be determined that the photovoltaic cell is working on the right side of the maximum power point at this time, and the duty cycle should be reduced at this time. If one of the two comparisons is greater than one and the other is less than, it can indicate that the photovoltaic cell is working near the maximum power point at this time, and the duty cycle is not adjusted.

Through the experimental simulation results, it can be seen that the method of pairwise comparison to determine the disturbance direction proposed in this paper has certain advantages. Its control logic includes every situation, and the tracking speed and tracking efficiency have a certain improvement compared with traditional methods.

First set the reference quantities $H$ and $Y$, [9] when $P_{k}>P_{k-1}, Y=1$, otherwise $Y=-1$. When $P_{k+l}>P_{k}, Y=1$ otherwise $Y=-1$. when $P_{k}=P_{k-1}, P_{k+l}=P_{k}, Y=0 . H=Y_{l}+Y_{2}$. The MPPT control process 
introduced in this article is as follows:

$$
\begin{aligned}
& \text { If } P_{k}>P_{k-1}, P_{k+1}>P_{k}, \text { then } H=2 \\
& \text { If } P_{k}>P_{k-1}, P_{k+1}<P_{k}, \text { then } H=0 \\
& \text { If } P_{k}<P_{k-1}, P_{k+1}<P_{k}, \text { then } H=-2 \\
& \text { If } P_{k}>P_{k-1}, P_{k+l}=P_{k} \text {, then } H=1 \\
& \text { If } P_{k}=P_{k-1}, P_{k+1}<P_{k}, \text { then } H=-1 \\
& \text { If } P_{k}=P_{k+1}, P_{k-1}=P_{k}, \text { then } H=0
\end{aligned}
$$

The above six situations do not include misjudgment. If a misjudgment occurs, it means that the system has made an error. Therefore, they are all judged as $H=0$, and the existing duty cycle remains unchanged [10].

This control method of changing the duty cycle based on the disturbance observation method can significantly reduce the oscillation of the output power of the photovoltaic cell at the maximum power point.

The duty cycle is sampled and the relationship between the power is compared and then an appropriate duty cycle is output. Finally, the output duty cycle is PID adjusted and the final duty cycle is output to the switching element in the Boost circuit.

The photovoltaic cell can output the maximum power, so that the cell always works near the maximum power point to achieve the purpose of tracking the maximum common power. The control principle diagram and control flowchart are shown in the Figure 8 and Figure 9.

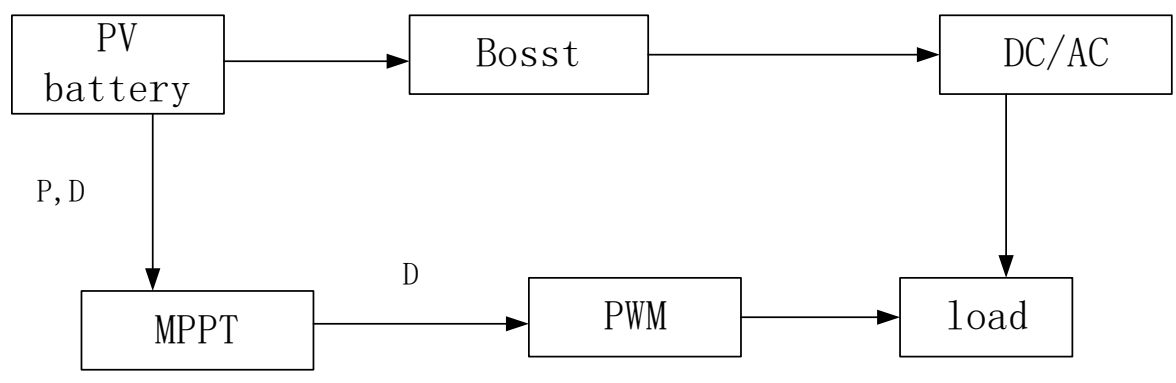

Fig. 8. Schematic diagram of duty cycle disturbance. 


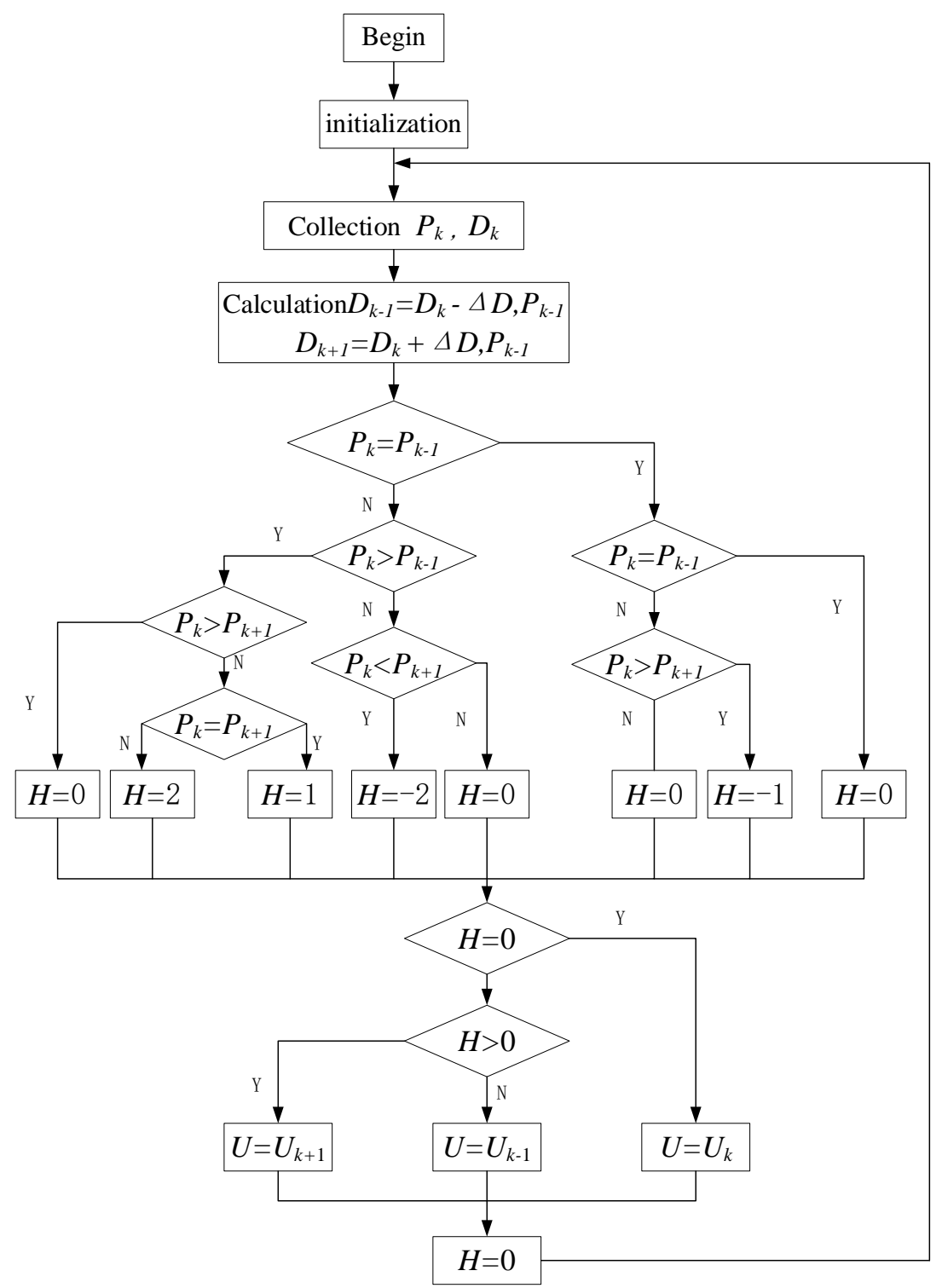

Fig. 9. Duty cycle disturbance control flowchart

The overall model of photovoltaic cells can be seen as a combination of several modules, first is the photovoltaic cell module, then the Boost circuit module, MPPT control module, and PWM pulse width modulation module. Combine various modules together to form the entire photovoltaic power generation system [11]. The simulation model is shown in the Figure10. 


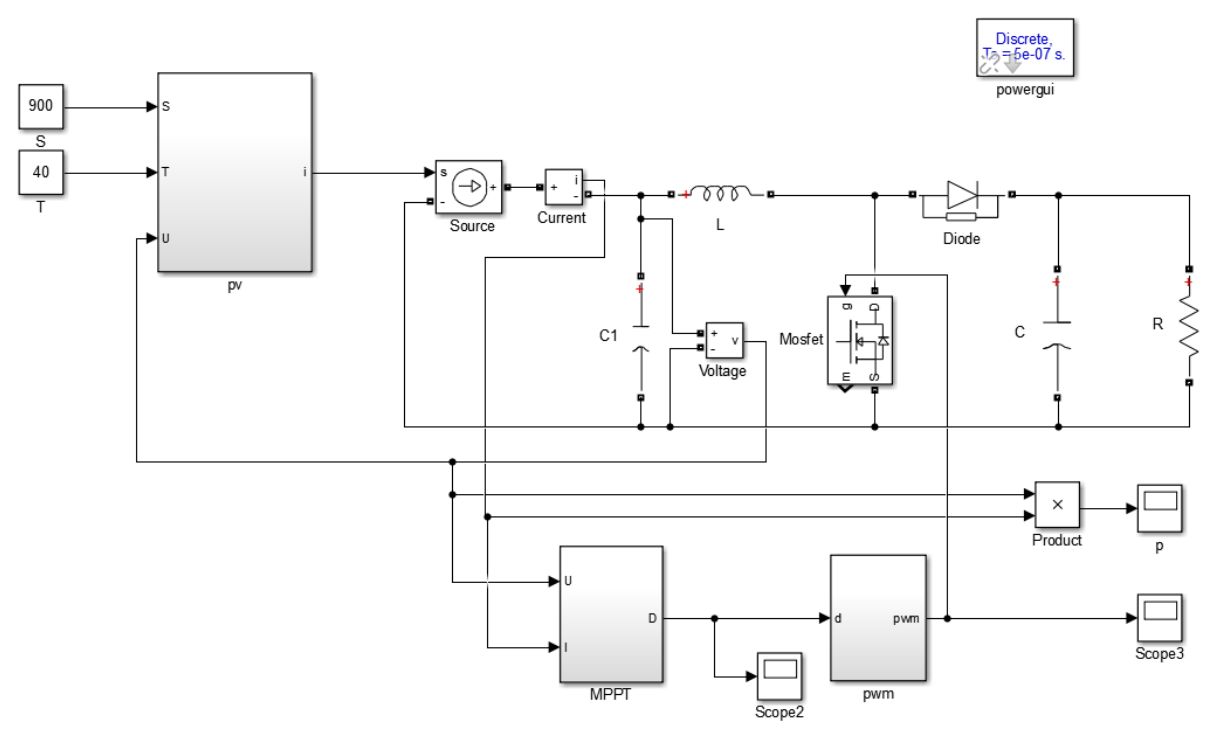

Fig. 10. Overall model of photovoltaic cells.

The power tracking results obtained by running the above model in Matlab/Simulink are shown in Figure 11 and Figure 12.

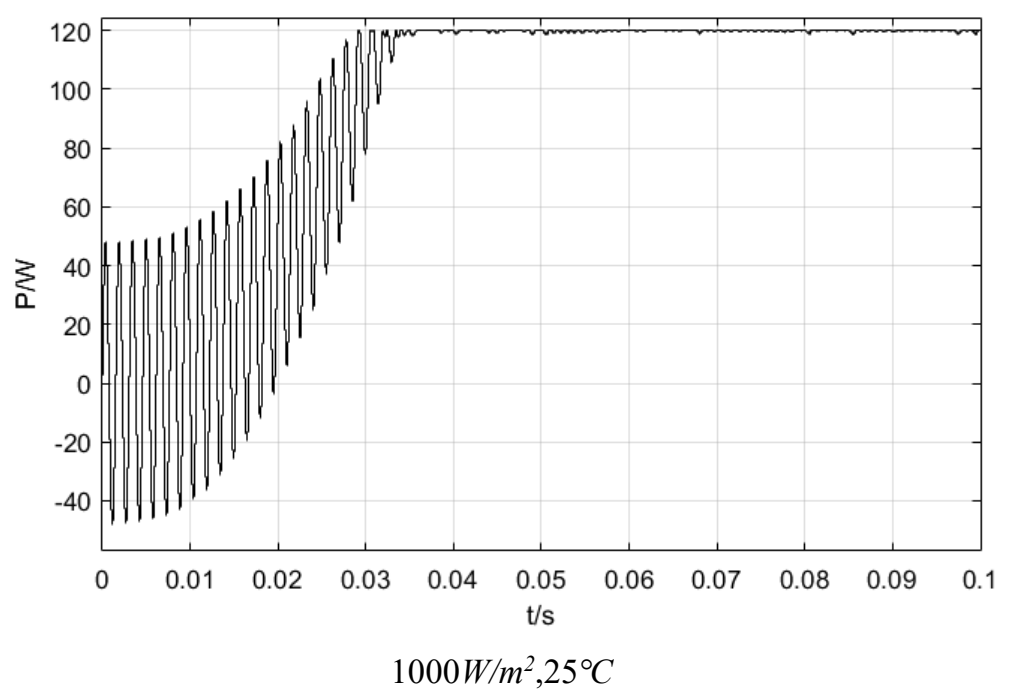

Fig. 11. Power tracking graph. 


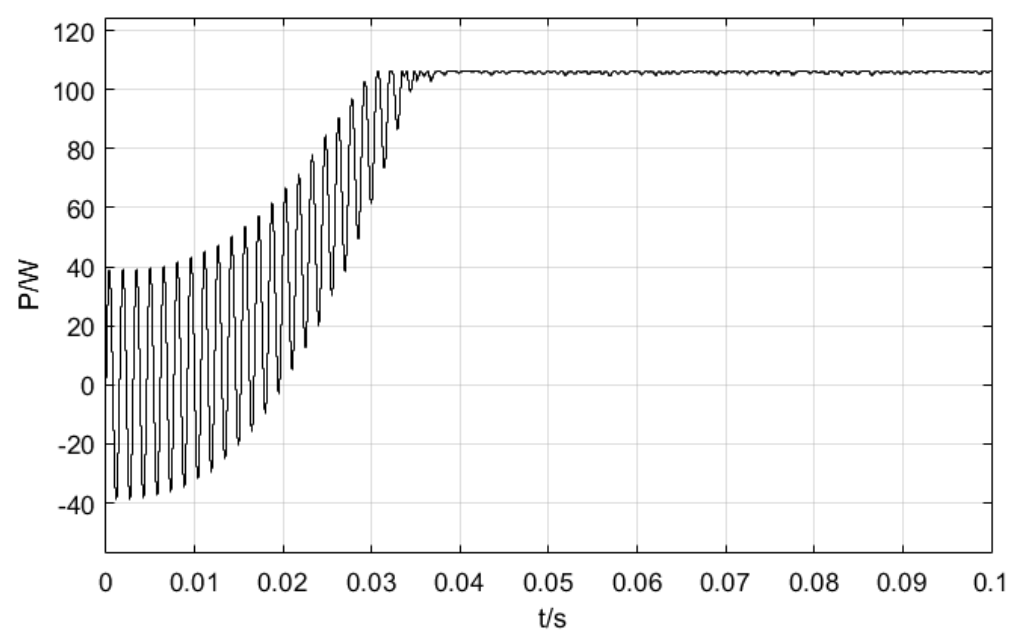

$900 \mathrm{~W} / \mathrm{m}^{2}, 25^{\circ} \mathrm{C}$

Fig. 12. Power tracking graph.

\section{Conclusion}

At present, there are many methods for maximum power tracking of photovoltaic systems. The traditional methods can no longer meet people's requirements due to their imperfect tracking efficiency and tracking accuracy. Therefore, this article proposes an improved disturbance observation method. And the feasibility of the method is verified through simulation experiments. This method avoids the problem of photovoltaic cells oscillating at the maximum power point, reduces power loss and increases the efficiency of power tracking. For the power tracking research of photovoltaic systems, A certain reference price.

\section{Acknowledgments}

The authors acknowledge financial support from the Natural Science Foundation of Educational Department of Liaoning Province (Grant: J2020053), and Technology Innovation Fund (Grant: 2020JJ26GX029) and would like to express many thanks to the support of Dalian Key Laboratory of Smart Micro-grid and Green Recycling Industry. 


\section{References}

[1] Lin Zhou., Jian Wu., Ke Guo.: Overview of the maximum power point tracking control method for photovoltaic arrays[J]. High voltage technology,2008(06):1145-1154.

[2] Liu F., Duan S., Liu B., et al.: A Variable Step Size INC MPPT Method for PV Systems[J]. IEEE Transactions on Industrial Electronics, 2008, 55(7):2622-2628.

[3] Koutroulis., Eftichios., Kalaitzakis., et al.: Development of a Microcontroller-Based Photovoltaic Maximum Power Point Tracking Control System[J]. IEEE Transactions on Power Electronics, 2001.

[4] Xuemei Yao.: Research on Optimal Control of Solar Energy (Photovoltaic) Application System[D]. Qingdao University, 2009.

[5] Xiangyu E., Jianjun Tan., Lei Zou., et al.: Improved photovoltaic MPPT algorithm based on disturbance observation method[J]. Journal of Hubei University for Nationalities (Natural Science Edition), 2018.

[6] Yibin Zheng.: Maximum power tracking and grid connection control method of photovoltaic power generation system[D]. Southwest Jiaotong University, 2016.

[7] Yidan Li., Duting Wang., Xianhui Zhu., Hongming Kang., Sanmin Wei.: Maximum power point tracking control based on adaptive duty cycle disturbance method[J]. Heilongjiang Electric Power,2015,37(02):102-104+115.

[8] Shengyun Jing.: New type of boost converter and its application in photovoltaic power generation system[D]. North China University of Technology, 2018.

[9] Bo Fang., Shuke Luo., Longyun Kang.: Research on MPPT and Simulation of Photovoltaic Duty Cycle Disturbance Control[J]. Renewable Energy,2013,31(10):5-9.

[10] Wu Yuwei., Shi Bin., Zhu Haiyong., Zhang Jialing.: Maximum power point tracking of photovoltaic cells based on variable step disturbance observation method[J]. Electrical Technology, 2014(06): 23-25.

[11] Huang Yao., Huang Hongquan.: Incremental conductance method to realize the maximum power point tracking control of photovoltaic system[J]. Modern Electronic Technology, 2008(22): 18-19. 